\title{
A Ventral Uncrossed Corticospinal Tract in the Rat
}

\author{
H. Lee Vahlsing and Earl R. Feringa ${ }^{1}$ \\ Departments of Neurology and Pathology, Veterans Administration, Ann Arbor, Michigan \\ 48105, and University of Michigan Medical Centers, Ann Arbor, Michigan 48104
}

Received February 12, 1980; revision received April 22, 1980

\begin{abstract}
By studying cross-section autoradiograms of the spinal cord with dark field microscopy we demonstrated a ventral uncrossed corticospinal tract in the rat. Corticospinal fibers were labeled by the slow axoplasmic flow of a minute volume of high specific activity tritiated proline injected directly into the motor sensory cortex. The uncrossed ventral corticospinal tract was small bur easily identifiable in the cervical region. More caudally the tract became less distinct and could not be traced below midthoracic levels. Only two corticospinal tracts were identified in this study: the well-known crossed dorsal corticospinal tract and the ventral uncrossed corticospinal tract described in this study.
\end{abstract}

The corticospinal tract in the rat has been described as an easily identified group of fibers situated in the most ventral portion of the dorsal white funiculus of the spinal cord $(1,3,5)$. Aside from a single abstract (2), we know of no report of ancillary corticospinal tracts in the rat spinal cord. Other reports indicate that the corticospinal tract in the rat spinal cord is completely crossed at the level of the caudal medulla oblongata and lies in the most ventral portion of the dorsal white columns.

In the course of study of transport of tritiated proline by corticospinal axons in the rat (6), we discovered a constant ventral corticospinal tract which was easily identified by dark field illumination of cross-section autoradiographs of the spinal cord.

\footnotetext{
1 This work was supported by the Veterans Administration Research Services, Ann Arbor, MI and San Diego, CA and by the Development Funds, Department of Pathology, University of Michigan Medical Center. The authors acknowledge the assistance of Ms. Linda Lee Austin (histology), Mr. Robert McKnight (photography), and Mrs. Diane Trakas and Barbara Reader (manuscript preparation). Reprint requests should be sent to Earl R. Feringa, M.D., Neurology Service (127), Medical Center, 3350 La Jolla Village Drive, San Diego, CA 92161.
} 


\section{MATERIALS AND METHODS}

Animals. Our animals are an inbred strain of albino rats derived from Wistar rats after 90 or more generations of brother-sister matings. They are isogeneic, as demonstrated by skin grafting experiments.

Surgical Procedures. Five 12-week-old females (approximately $125 \mathrm{~g}$ weight) were anesthetized with ether and immobilized in a stereotaxic apparatus. The skull was exposed and a $1-\mathrm{mm}$ burr hole was made $2.5 \mathrm{~mm}$ posterior to the coronal suture and $2.5 \mathrm{~mm}$ lateral to the sagittal suture directly above the area of cerebral cortex containing the cell bodies that give rise to the corticospinal tract in the rat $(1,4)$. Using a micromanipulator and a short-bevel, 30-gauge needle, $50 \mu \mathrm{Ci}$ tritiated proline in $2.5 \mu \mathrm{l}$ lactated Ringer's solution was slowly (30 to $60 \mathrm{~s}$ ) injected at a depth of $1 \mathrm{~mm}$ beneath the pial surface. After the animal and the injecting apparatus were kept immobile for $1 \mathrm{~min}$, the needle was withdrawn and the scalp sutured.

Evaluation. Four weeks later, after slow axoplasmic transport had labeled the corticospinal tract (6), each animal was anesthetized and killed by Formalin perfusion. The brain and spinal cord were removed. Cross sections of the brain were sampled every $2 \mathrm{~mm}$; cross sections of the spinal cord were sampled every $10 \mathrm{~mm}$. Sections were cut at $6 \mu \mathrm{m}$, mounted on slides, and dipped in NTB-2 emulsion. They were exposed 3 weeks in a light-tight box with a $\mathrm{CO}_{2}$ atmosphere and developed for $30 \mathrm{~s}$ in developer diluted 1:2 with water. The autoradiograms were examined by dark-field microscopy.

\section{RESULTS}

The cross-section autoradiograms at the level of the injection site showed dense silver grains directly over the region of the injection site and adjacent cerebral cortex (Fig. 1A). Exiting axons were labeled in the corpus callosum, terminating in the contralateral homologous cortex. The ipsilateral internal capsule was heavily labeled. Some of the labeled axons extended into the ipsilateral ventral thalamic nucleus and the reticular nucleus of the thalamus.

At the level of the mesencephalic-metencephalic junction (Fig. 1B), and in the metencephalon (Fig. 1C), only one descending corticospinal projection was labeled and this was on the ipsilateral side. In the far caudal portions of the medulla oblongata (Fig. 1D), most of this tract decussated dorsally and medially to assume positions in the ventral part of the dorsal white funiculus. However, a small (10\%) but significant number of fibers of this tract continued uncrossed in a position just lateral to the ventral median fissure. This uncrossed tract was visible as a discrete fiber bundle in the high cervical region (Fig. 1E). It became more diffuse and contained 

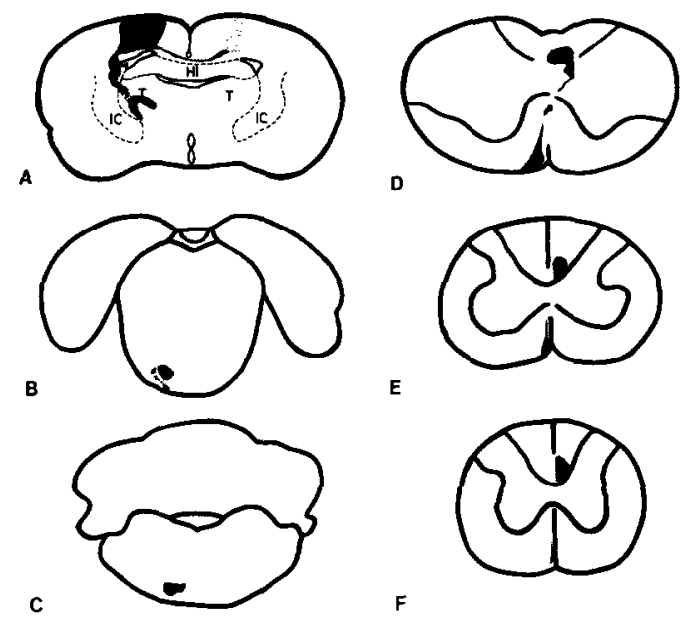

FIg. 1. Line drawings indicating the loci of projections from the sensory motor cortex identified by dark-field examination of autoradiograms 2 weeks after the intracortical injection of tritiated proline. A-Shows the distribution of radioisotope at the site of injection. Note spread across the corpus callosum to the contralateral homologous cortex, label in the lateral thalamus, and label in the internal capsule. IC, internal capsule, T, thalamus HI, hippocampus. B-taken at the level of the mesencephalic-metencephalic junction demonstrating a single corticospinal tract in the lower pons. $\mathrm{C}$-demonstrates a single ipsilateral corticospinal tract. D-at the caudal-most level of the medulla, fibers from the pyramid decussate upward forming the previously described dorsal crossed corticospinal tract. A few fibers, however, remain behind in their ventral position. E-shows both the dorsal crossed and ventral uncrossed corticospinal tracts as they are identified in the high cervical region. F-a section through the high thoracic region where the dorsal crossed corticospinal tract remains easily identifiable but the ventral uncrossed corticospinal tract is beginning to decrease in size.

fewer fibers in the lower cervical segments and in the thoracic spinal cord (Fig. 1F); it could not be traced below the midthoracic level by this autoradiographic technique nor could we identify the terminations of these axons in the cervical spinal cord gray matter.

In other experiments $(7,10)$ we observed autoradiographs of cross sections of the cervical spinal cords of more than 50 similarly labeled rats. In each case, both dorsal crossed corticospinal tracts and ventral uncrossed corticospinal tracts were noted at cervical levels (Fig. 2).

\section{DISCUSSION}

In 1966 Goodman et al. (2), reported in abstract several distinct corticospinal pathways in the albino rat. They used the Nauta silver method to stain preterminal degenerating axons at selected times after cortical surgical lesions. They reported that unilateral frontoparietal cortical destruc- 


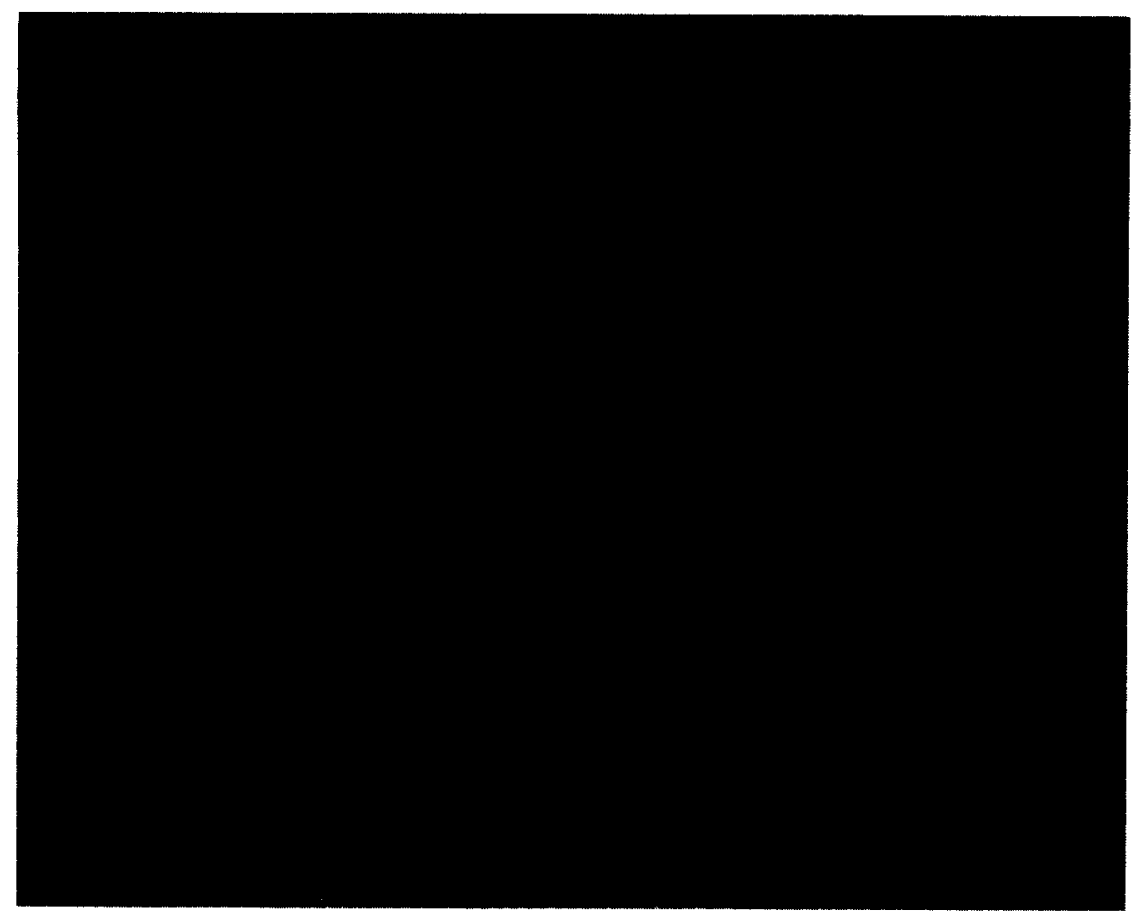

FIG. 2. A-photograph of the cervical spinal cord showing both the dorsal crossed and ventral uncrossed corticospinal tracts, by the double exposure technique of superimposition of dark-field and bright-field images $(7) \times 28.2$. B - photographic enlargement showing detail of ventral corticospinal tract.

tion resulted in degeneration which could be found in three separate tracts: (i) the dorsal funicular tract bilaterally, (ii) a lateral corticospinal tract bilaterally, and (iii) an ipsilateral ventral corticospinal tract. The dorsal 
tract contralateral to the lesion was largest and the only tract which reached sacral levels. They reported that the ipsilateral dorsal and ipsi- and contralateral lateral tracts extended to the L5 level, whereas the ipsilateral ventral corticospinal tract was not present below C7. Goodman (personal communication) studied very large adult albino male rats from a breeding colony.

We were able to confirm the presence of the crossed dorsal corticospinal tract and the uncrossed ventral corticospinal tract, but we saw no labeled lateral corticospinal tract on either side, and no ipsilateral dorsal corticospinal tract. This apparent discrepancy may be the result of rather different labeling techniques and differing evaluation procedures.

Goodman's surgical ablation labeling technique runs the risk of inadvertent injury to other regions (i.e., the contralateral cerebral hemisphere) with the result of overstaining in the spinal cord. Conversely, our cortical injection technique is very localized in its effect and runs the possible risk of labeling a much smaller cortical region with resultant demonstration of fewer corticospinal axons in the spinal cord.

We believe the labeled uncrossed ventral corticospinal tract which we describe represents first-order corticospinal projections. Contrary to other reports in the literature, we found no evidence that the radioactive proline label was passed across the synapse to the next order neuron. Specifically, in this experiment, axonal transport carried the label via the corpus callosum to the contralateral cortex, but we never found label in the axons exiting from this cortex in the internal capsule on that side. Similarly, ipsilateral corticothalamic axons were labeled as were their terminals in the ventral thalamus. No labeled projections from the thalamus were seen. Thus we lack any evidence that this radioisotopic label was passed from one neuron to the next.

The evaluation procedure we used to study the corticospinal projections in the spinal cord was very different from that of Goodman et al. (2). The brightly shining silver grains over a group of labeled fibers is easily identified on autoradiographic cross sections of the spinal cord studied with dark-field illumination. On the other hand, scattered longitudinal fibers, not grouped together as a fascicle, would be identified only as isolated silver grains. As such, they would be difficult or impossible to separate from background. For these reasons we are very confident that we identified the tracts described. It is possible, however, that one or more other diffuse corticospinal tracts in other loci could be missed. Other investigators, using variations of the Nauta technique, however, have not noted other corticospinal tracts (3).

Goodman et al. (2) found the tracts they described in very large older male rats. This report details findings in young female rats. However, the 
same tracts are invariably found in old or young, male or female rats from our colony.

\section{REFERENCES}

1. D'Amato, C. J., AND S. P. Hicks. 1978. Normal development and posttraumatic plasticity of corticospinal neurons in rats. Exp. Neurol. 60: 557-569.

2. Goodman, D. C., L. E. JarRaRd, AND J. F. Nelson. 1966. Corticospinal pathways and their sites of termination in the albino rat. Anat. Rec. 154: 462.

3. Hicks, S. P., AND C. J. D' AMATo. 1975. Motor-sensory cortex-corticospinal system and developing locomotion and placing in rats. Am. J. Anat. 143: 1-43.

4. HICKs, S. P., AND C. J. D' AMATo. 1976. Locating corticospinal neurons in mature, infant, and malformed (radiation) rats with retrograde axonal transport of horseradish peroxidase. Soc. Neurosci. Abstr. 2: 123.

5. Ranson, S. W. 1913. The fasciculus cerebro-spinalis in the albino rat. Am. J. Anat. 14: 411-424.

6. Vahlsing, H. L., E. R. Feringa, and R. B. Hirschl. 1978. Axonal transport of tritiated proline in the corticospinal tract of the rat spinal cord. Soc. Neurosci. Abstr. 4: 38.

7. Feringa, E. R., L. M. Shuer, H. L. Vahlsing, and S. W. Davis. 1977. Regeneration of corticospinal axons in the rat. Ann. Neurol. 2: 315-321.

8. Feringa, E. R., T. F. Kowalski, H. L. Vahlsing, and R. A. Frye. 1979. Enzyme treatment of spinal cord transected rats. Ann. Neurol. 5: 203-206.

9. KowAlSKI, T. F., H. L. VAHLSING, AND E. R. FerINGA. 1979. Lidase treatment of spinal cord transected rats. Ann Neurol. 6: 78-79.

10. Feringa, E. R., K. R. Nelson, H. L. Vahlsing, and R. C. Dauser. 1979. Spinal cord regeneration in rats made immunologically unresponsive to CNS antigens. J. Neurol. Neurosurg. Psychiat. 42: 642-648. 